\title{
Coronavirus disease and the cardiovascular system: a narrative review of the mechanisms of injury and management implications
}

\author{
Maria Vega Brizneda ${ }^{1}$, Agam Bansal ${ }^{2}$, Vardhmaan Jain ${ }^{2}$, Samir Kapadia ${ }^{1}$, Lars G. Svensson ${ }^{3}, \operatorname{Venu}_{\text {Menon }}{ }^{1}$, \\ Paul Cremer ${ }^{1}$, Grant Reed ${ }^{1}$, Penelope Rampersad ${ }^{1}$, Richard Grimm ${ }^{1}$, Brian P. Griffin ${ }^{1}$, Bo Xu ${ }^{1}$ \\ ${ }^{1}$ Robert and Suzanne Tomsich Department of Cardiovascular Medicine, Sydell and Arnold Miller Family Heart, Vascular and Thoracic Institute, \\ Cleveland Clinic Foundation, Cleveland, OH, USA; ${ }^{2}$ Department of Internal Medicine, Cleveland Clinic, Cleveland, OH, USA; ${ }^{3}$ Department \\ of Thoracic and Cardiovascular Surgery, Sydell and Arnold Miller Family Heart, Vascular and Thoracic Institute, Cleveland Clinic Foundation, \\ Cleveland, OH, USA \\ Contributions: (I) Conception and design: B Xu, MV Brizneda, A Bansal, V Jain; (II) Administrative support: MV Brizneda, B Xu; (III) Provision of \\ study materials or patients: MV Brizneda, A Bansal, V Jain, B Xu; (IV) Collection and assembly of data: MV Brizneda, A Bansal, V Jain, B Xu; (V) \\ Data analysis and interpretation: All authors; (VI) Manuscript writing: All authors; (VII) Final approval of manuscript: All authors. \\ Correspondence to: Bo Xu, MB, BS (Hons), FRACP, FACC, FASE. Section of Cardiovascular Imaging, Robert and Suzanne Tomsich Department of \\ Cardiovascular Medicine, Sydell and Arnold Miller Family Heart, Vascular and Thoracic Institute, Cleveland Clinic, 9500 Euclid Avenue, Desk J1-5, \\ Cleveland, OH 44195, USA. Email: xub@ccf.org.
}

\begin{abstract}
Coronavirus disease (COVID-19), first identified in Wuhan, China, in December 2019, is now a pandemic, having already spread to 188 countries, with more than 28,280,000 infections worldwide. Severe Acute Respiratory Syndrome Coronavirus 2 (SARS-CoV-2) is the responsible infectious agent, and similar to other human coronaviruses, uses membrane-bound angiotensin-converting enzyme 2 (membrane-bound ACE2) for entry into the host cells. COVID-19 has important cardiovascular implications, especially for patients with pre-existing cardiovascular co-morbidities, potentially mediated through several mechanisms, including direct myocardial injury, worsening of those pre-existing cardiovascular co-morbidities, and adverse cardiovascular effects of potential therapies for COVID-19. The disease is causing a significant burden on health systems worldwide. Elective surgeries and procedures were postponed for a considerable period of time, and many patients with known cardiovascular disease (CVD) risk factors presented late to hospitals, for fear of contracting COVID-19, with serious adverse consequences. Significant negative impact on a population level is highlighted by prolonged isolation, decreased exercise and physical activity, and higher levels of depression and anxiety, all predisposing to elevated cardiovascular risk. This article provides a timely overview of COVID-19 and its impact on the cardiovascular system, focusing on the pathogenesis, potential adverse cardiovascular events, the potential treatment options, protection for health care providers and patients, and what the cardiovascular community could do to mitigate the impact of COVID-19.
\end{abstract}

Keywords: Coronavirus disease; COVID-19; cardiovascular system; cardiovascular disease (CVD); membranebound ACE2

Submitted Sep 12, 2020. Accepted for publication Dec 21, 2020.

doi: $10.21037 / \mathrm{cdt}-20-779$

View this article at: http://dx.doi.org/10.21037/cdt-20-779

\section{Introduction}

The novel coronavirus disease (COVID-19), was declared a global pandemic on March 11, 2020 (1). First identified in Wuhan, China, in December 2019 (2), is now present in
188 countries (3). As of October 10, 37,278,000 cases were confirmed, with 1,075,000 deaths globally (3). The disease has spread across the United States (US), which tops the global tally with >7.3 million confirmed cases and 215,000 deaths, followed by India and Brazil, with 7,730,000 and 


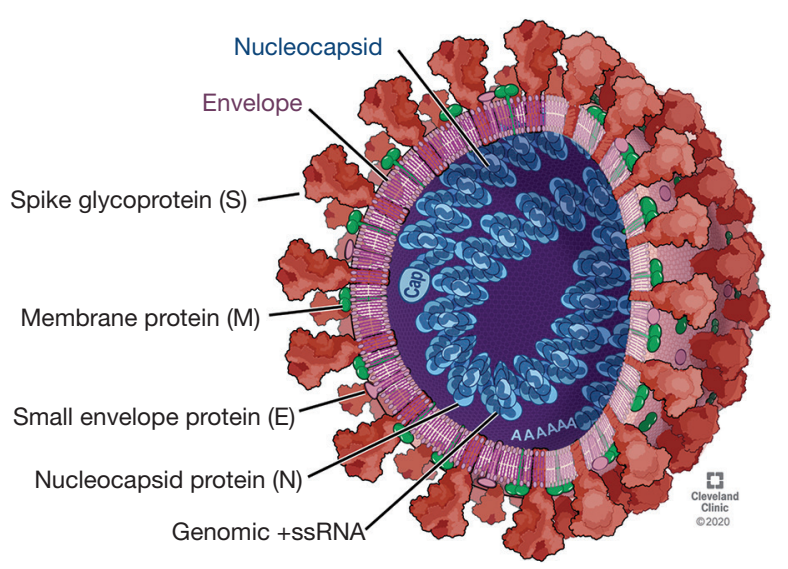

Figure 1 Structure of the Severe Acute Respiratory Syndrome Coronavirus 2 (SARS-CoV-2) virus. RNA, ribonucleic acid.

7,054,000 confirmed cases respectively (3).

The agent responsible is the Severe Acute Respiratory Syndrome Coronavirus 2 (SARS-CoV-2), the third virus of the Coronaviridae family causing outbreaks in humans over the past two decades (4). The infection route is not well understood. The dominant hypothesis is a zoonotic infection transmitted from bats to an intermediate host (presumably the Malayan pangolin) and postulated contamination of food consumed by humans in a Wuhan market (5). After the exposure, infected patients presented with pneumonia-like syndromes to local hospitals, and subsequently, person-toperson spread via droplets contributed to the dissemination to other areas (2). Droplet spread occurs when fluids in the mucosa containing the virus travel short distances through the air by sneezing/coughing (6). Inanimate objects and surfaces are also reservoirs of contaminated droplets that can infect after hand contact, followed by touching the mouth, nose, or eyes (6). Airborne dissemination and fecaloral transmission are also described (6). Fecal samples have been found to contain the virus, and $15 \%$ of patients debut with gastrointestinal symptoms (7). We present the following article in accordance with the Narrative Review reporting checklist (available at http://dx.doi.org/10.21037/cdt-20-779).

\section{Pathogenesis}

SARS-CoV-2 is a betacoronavirus with a positive singlestranded Ribonucleic Acid (RNA) envelope in the same genera as the Middle East Respiratory Syndrome (MERS$\mathrm{CoV}$ ) and the Severe Acute Respiratory Syndrome (SARS$\mathrm{CoV}$ ) viruses (8). SARS-CoV-2 shares $96 \%$ of its sequence with two bat-derived SARS-like coronaviruses (BatCoV and RaTG13), $80 \%$ of its sequence with SARS-CoV, and $50 \%$ of its sequence with MERS-CoV $(4,9)$. Like SARS-CoV, its components include the spike glycoprotein (S-protein), envelope (E), nucleocapsid $(\mathrm{N})$ and membrane $(\mathrm{M})$ proteins (Figure 1) (4). S-protein binds to membrane-bound angiotensin-converting enzyme 2 (membrane-bound ACE2), and along with a transmembrane protease serine 2 receptor (TMPRSS2) in host cells, are essential for viral fusion to the cellular membrane $(9,10)$. An alternate entry pathway is clathrin-dependent and independent endocytosis (9). Upon cytoplasm entry, viral RNA is released to start replication. Translation of structural polypeptide chains initiates, and proteolysis occurs to create a replication-transcription complex with the catalytic enzyme RNA-dependent RNA polymerase. The synthesis and assembly of viral RNA into enveloped glycoproteins begins in the endoplasmic reticulum. Finally, after packing the vesicles containing the virus, the virion is released (4).

ACE2 is present in the lungs, heart, kidneys, intestine, and vascular endothelium, with higher expression in the lungs and heart (9). The vast majority of pulmonary membrane-bound ACE2 is expressed in Type II alveolar cells (approximately 83\%), serving as a reservoir for the infection (10). The characteristics and the presence of high levels of regulatory genes in the type II alveolar cells constitute an ideal media for the virus to complete its life cycle, assembly, and replication (10). With the binding of the virus to membrane-bound ACE2 and other systemic factors, such as the cytokine storm, patients are highly predisposed to injury, sepsis, and acute respiratory distress syndrome (ARDS) $(4,9)$.

The cardiovascular effects of COVID-19 are mediated through multiple mechanisms. Direct cardiotoxicity, as exemplified in prior influenza outbreaks and other coronavirus strains (MERS-CoV and SARS-CoV), can occur (11). ACE2 is an essential regulator of cardiac function. Its primary role is catalyzing the reaction that converts angiotensin I into angiotensin, and subsequently controlling blood pressure via vasoconstriction $(9,12)$. Also, it counteracts the consequences of excessive activity of the renin-angiotensin system (RAS) by mitigating the effects of angiotensin II (Figure 2) (12). In conditions such as hypertension (HTN) and heart failure (HF), the RAS is hyperactive, with low expression/availability of cardiovascular membrane-bound ACE2. There is a hypothesis of worsening cardiovascular disease (CVD) due to the further binding of SARS-CoV2 (9). Furthermore, 


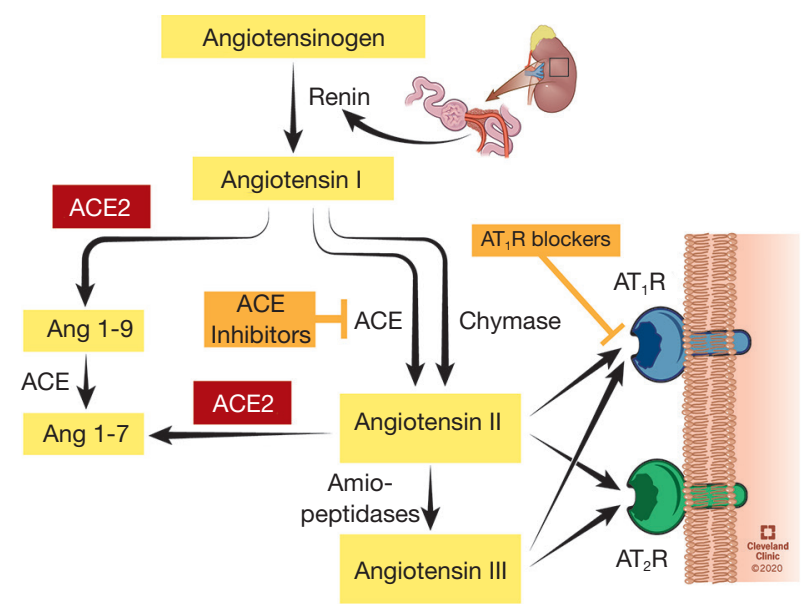

Figure 2 Diagram illustrating the angiotensin-converting enzyme 2 (ACE2) pathway, which is important for the pathogenesis of COVID-19. ACE, angiotensin-converting enzyme; ACE2, angiotensin-converting enzyme 2; Ang 1-9, angiotensin 1-9; AT1R, angiotensin II type 1 receptor; AT2R, angiotensin II type 2 receptor. the viral-induced systemic inflammatory response may exacerbate underlying CVD, predisposing to premature atherosclerotic plaque activation, and rupture (13). Another relevant effect to consider is the experimental therapies that are known to be, or potentially associated with cardiovascular adverse effects (discussed below).

COVID-19 is causing a significant burden on health systems worldwide. Elective surgeries and procedures are being postponed. In this context, some patients with known CVD may not be treated promptly (14). Finally, serious consequences impacting populations include prolonged isolation, decreased exercise, deficient diets, and higher levels of depression and anxiety, all predisposing to elevated cardiovascular risk (Figure 3).

\section{COVID-19 and cardiovascular system}

China and Italy reported high prevalence rates of CVD and death in the current COVID-19 pandemic (5). A

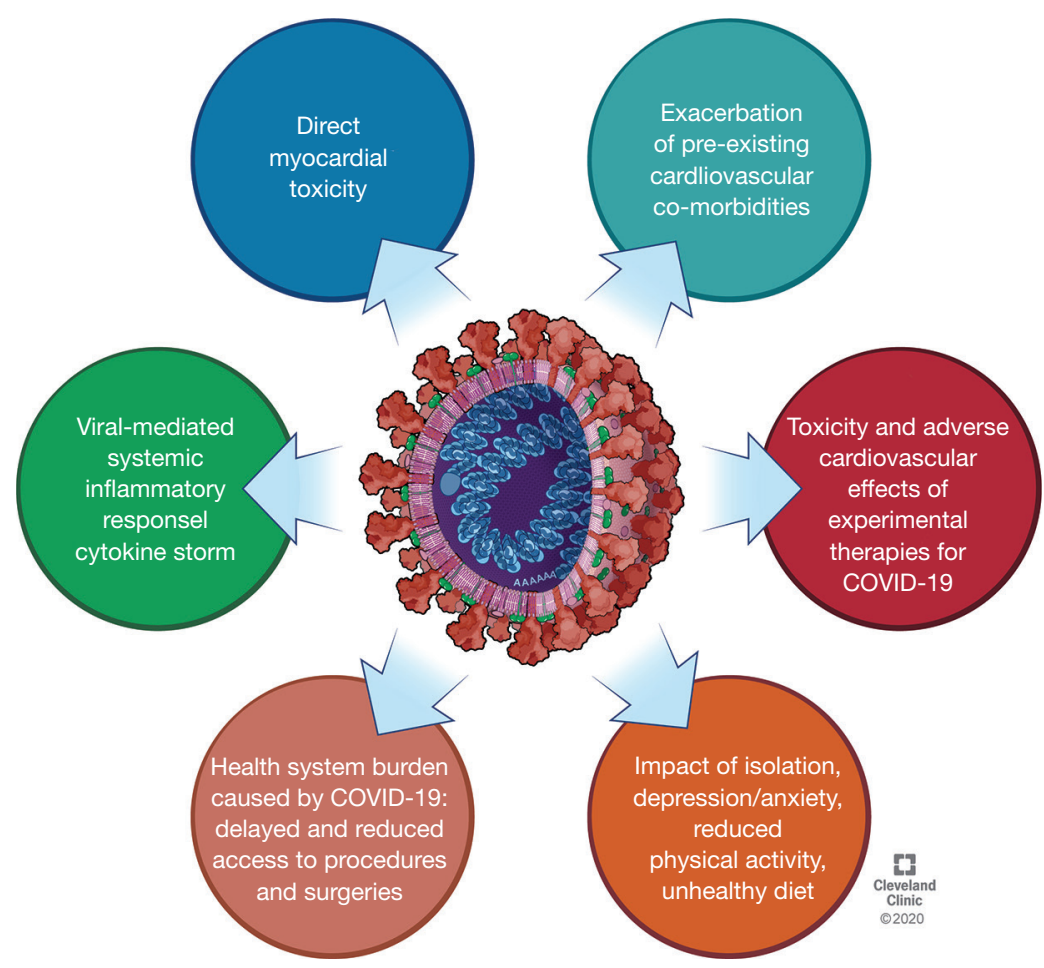

Figure 3 he cardiovascular effects of COVID-19 are mediated through multiple mechanisms. 
recent single-center study of 416 hospitalized COVID-19 patients noted that cardiac injury occurred in $19.7 \%$ of patients (commonly the elderly and those with pre-existing cardiovascular comorbidities) (15). It was associated with higher levels of troponin and inflammatory markers with a 3 to a 4-fold elevated risk of all-cause mortality, both from the time of symptom onset and admission (15). In a retrospective cohort of 191 COVID-19 patients, the prevalence of HTN, diabetes mellitus (DM), and CVD were reported to be $30 \%, 19 \%$, and $8 \%$, respectively, with even higher rates observed in those requiring intensive care unit (ICU), and those who died (13). In comparison, Middle East Respiratory Syndrome (MERS) and Severe Acute Respiratory Syndrome (SARS) were reported having an associated prevalence of DM, ranging between $11 \%$ to $50 \%$, which was associated with increased mortality $(16,17)$.

While these reports aid in understanding the CVD burden of COVID-19, the lack of external validity needs to be considered. Epidemiologically, the Asian population generally has a lower prevalence of cardiovascular risk factors compared to the Western population (18). Early US and European data have recently become available. In a single center case series of 21 critically ill COVID-19 patients in Washington state, $86 \%$ of the cohort had comorbidities (19). Chronic kidney disease and congestive $\mathrm{HF}$ were the most common (47.6\%, and 42.9\%, respectively) (19). Cardiomyopathy developed in $33 \%$ of the patients, and the overall mortality was $67 \%$ (19). A prospective observational cohort study in the United Kingdom (UK), enrolling 16,749 hospitalized patients from 166 centers, is the largest study in Europe to date, characterizing the clinical features of critically ill COVID-19 patients (20). Similarly, comorbidities were commonly present (53\% of the cohort) (20). CVD was the most common comorbidity (29\%), followed by DM and nonasthmatic pulmonary diseases (19\% each) (20). The presence of comorbidities were associated with higher mortality rates (33\% in the comorbidity group) (20) (Figure 4).

\section{Myocarditis and myocardial injury}

COVID-19 has been associated with myocardial injury, as evidenced by elevated cardiac biomarkers (15). In a cohort of 416 confirmed cases from China, patients with confirmed cardiac injury had higher levels of $\mathrm{N}$-terminal pro-B-type natriuretic peptide (NT-proBNP) \{median [interquartile range (IQR)], 1,689 [698-3,327] vs. 139 [51-335] pg/mL\}, high sensitivity Troponin I (hs-TNI) [median (IQR), 0.19 $(0.08-1.12) v s .<0.006(<0.006-0.009) \mu \mathrm{g} / \mathrm{L}]$, creatine
kinase-MB (CK-MB) [median (IQR), 3.2 (1.8-6.2) vs. 0.9 $(0.6-1.3) \mathrm{ng} / \mathrm{mL}]$, and other acute-phase proteins such as C-reactive protein (CRP), and procalcitonin (15). Similarly, in 138 hospitalized patients from China, $7.2 \%$ of the patients had elevated cardiac biomarkers, electrocardiographic changes, or echocardiographic abnormalities. $22 \%$ of the cohort required ICU admission (21). Another retrospective study from China reported that hs-TNI levels were elevated $>99$ th percentile of reference range more frequently in fatal cases (13). The summative findings from several Chinese studies reported that the severity of myocardial injury was significantly greater in patients needing ICU admission and in those who died $(2,13,22)$.

Prior experiences from the MERS outbreak, showed that direct viral-mediated acute myocarditis could manifest as myocardial edema, and localized wall motion abnormalities (23). However, early pathological reports from COVID-19 cases, reported scarce mononuclear tissue infiltrates and a lack of substantial viral-induced myocardial damage, arguing against this being a dominant mechanism (23). The other suggested mechanism for myocardial injury is immune dysregulation and cytokine storm in the setting of systemic viremia (15). Huang et al. and Shi et al. reported higher levels of CRP, leucocytes, methemoglobinemia, elevated interleukin (IL)-2, IL-7, IL-10, and granulocyte-colony stimulating factor in hospitalized COVID-19 patients $(8,15)$. The argument for cytokine storm is also supported by reports of patients with fulminant myocarditis and severely depressed left ventricular ejection fraction, who were treated successfully with high-dose immunoglobulins, steroids, antiviral therapy, and supportive care (24).

\section{Acute coronary syndrome}

There are no extensive published studies reporting Type I acute myocardial infarction (AMI) directly caused by COVID-19 as of May 13, 2020. It should be noted that prior reports have shown that the systemic inflammatory response in the context of acute viral illnesses can trigger plaque destabilization and subsequently increased risk of acute coronary syndrome (25). Kwon et al. showed a 6-fold increased risk of AMI within 7 days of an influenza-related illness compared to control cases and a 2.8-fold increased risk associated with non-influenza viral diseases (25). Also, it is interesting to note that these are a few reported cases of COVID-19 presenting with symptoms mimicking AMI without significant obstructive coronary lesions $(24,26)$. These cases may result from an underlying cytokine storm or reflect a sympathetic overdrive mediated 


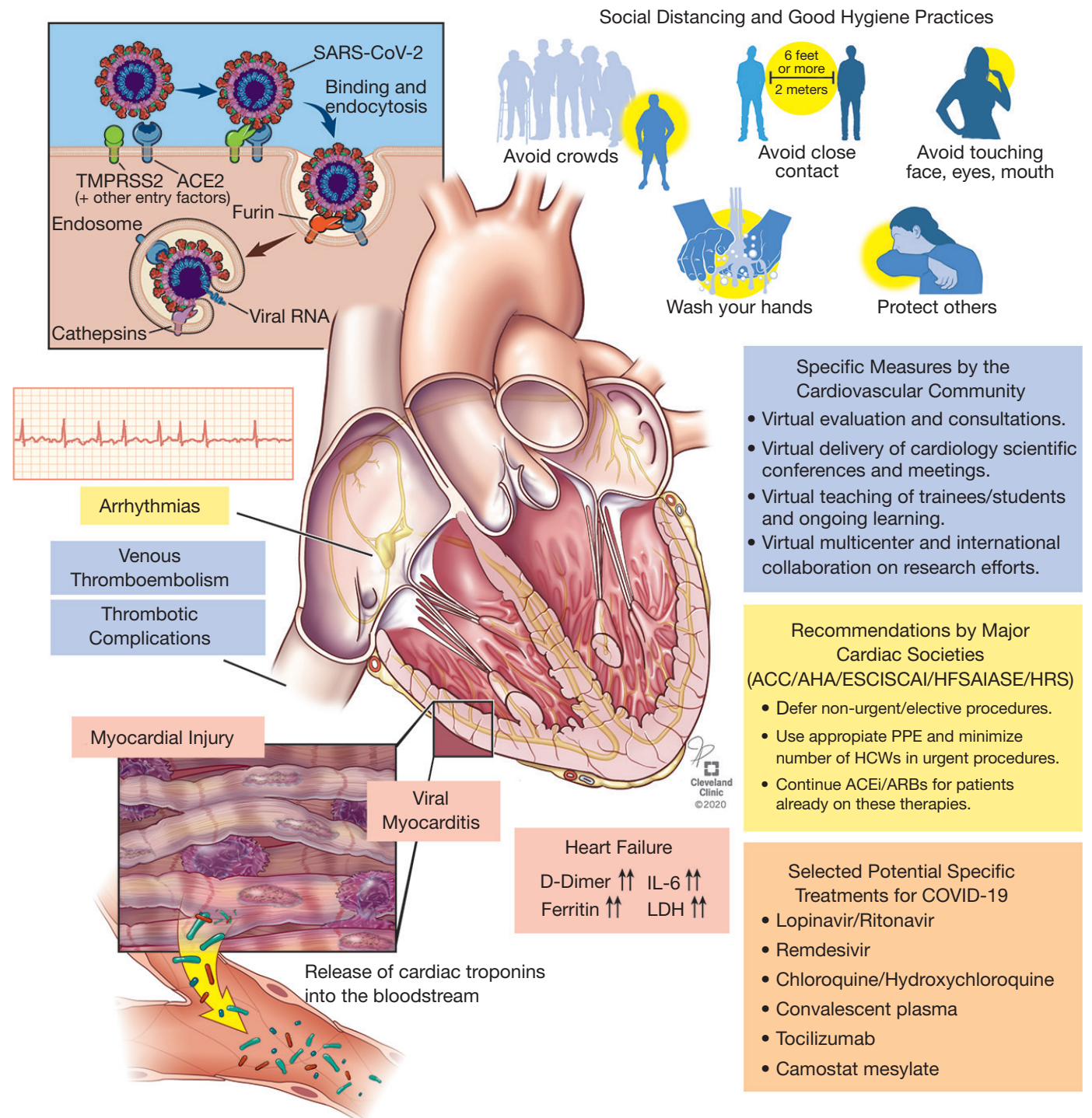

Figure 4 Central figure illustrating the impact of COVID-19 on the cardiovascular system, as well as the general and specific measures to combat COVID-19. SARS-CoV2, Severe Acute Respiratory Syndrome Coronavirus 2; TMPRSS2, transmembrane protease serine 2

Receptor; ACE2, angiotensin-converting enzyme 2; RNA, ribonucleic acid; ACC, American College of Cardiology; AHA, American Heart Association; ESC, European Society of Cardiology; SCAI, Society for Cardiovascular Angiography and Interventions; ASE, American Society of Echocardiography; HRS, Heart and Rhythm Society; PPE, personal protective equipment; HCWs, healthcare workers; COVID-19, coronavirus disease; ACEi/ARBs, angiotensin receptor inhibitors/angiotensin receptor blockers; IL, interleukin.

stress cardiomyopathy/Takotsubo-like pattern, and deserves further investigation.

\section{Arrbytbmia}

The data surrounding the prevalence of electrophysiological abnormalities in COVID-19 patients are not robust. In two
Chinese cohorts (138 and 137 patients), tachyarrhythmias were reported in $7 \%$ to $16 \%$ of hospitalized patients and were more common in patients needing ICU admission $(21,27)$. Prevalence and description of the specific subtypes of arrhythmias are under-reported at this stage. Current data support that electrocardiographic changes consistent with ischemia in the setting of elevated biomarkers may be 
indicative of cardiac injury and/or myocarditis (15).

\section{Heart failure, shock and heart transplantation}

A study of 191 hospitalized patients from two large tertiary hospitals in China, reported HF in $52 \%$ of the fatal cases, and $12 \%$ in the discharged patients. A significant proportion of these patients were complicated by cardiogenic shock (13). A substantial number of patients with underlying compromised cardiac function may also be at risk of developing acute rightsided HF (13). The same study also reported that 38 of 191 patients $(20 \%)$ developed septic shock, a universally fatal outcome (13). The clinician should therefore maintain a high degree of suspicion for multifactorial shock.

Consideration of heart transplant in the setting of severe COVID-19 cardiomyopathy needs to be further studied. While it is known to be a viable treatment option in cases of acute myocarditis, early reports of two heart transplant patients presenting with COVID-19, raise concerns of potential allograft rejection in the setting of an infected donor (28). Both cases were treated with high-dose immunoglobulins and corticosteroids, and were eventually discharged (28). Strict protocols regarding donor exposure and pre-transplant evaluation may conceivably render heart transplantation a viable management option for select patients.

Extracorporeal membrane oxygenation (ECMO), may be offered as a therapeutic option in select cases, according to the World Health Organization (WHO) (29). Veno-venous $(\mathrm{V}-\mathrm{V})$ ECMO is indicated for refractory ARDS $(29,30)$. In the context of cardiovascular complications causing cardiogenic shock, such as severe myocarditis, arrhythmias, significant pericardial effusions, and hemodynamically significant thrombotic complications, veno-arterial ( $\mathrm{V}-\mathrm{A})$ ECMO may be considered (30,31). However, even with the most advanced supportive measures, the prognosis of certain severe cases remain poor (32). The decision to initiate ECMO therapy should take into account of factors, such as patient safety, and experience and expertise of the individual center $(30,31)$.

\section{Thrombotic disease}

Emerging data suggest a higher risk of thrombotic and thromboembolic events associated with COVID-19 (33). A combination of prothrombotic factors (e.g., hyperinflammation, mechanical ventilation, the presence of central venous catheters, and prolonged immobilization), and hemostatic abnormalities (e.g., thrombocytopenia, increased D-dimer levels), likely play important roles (34). Patients with COVID-19 infections, are prone to pulmonary embolism (PE) (34). In a French case series of 107 patients with a confirmed diagnosis of COVID-19 admitted to the ICU, 20.6\% had PE. They found that compared to the hospitalized patients who tested negative for COVID-19, during a similar time interval, the frequency of $\mathrm{PE}$ in the COVID-19 series, was significantly higher $[20.6 \%$ vs. $6.1 \%$; absolute increased risk, 14.4\% (95\% CI, 6.1-22.8)] (35). In a Chinese study of 81 critically ill patients in the ICU, the incidence of lower extremity venous thromboembolism (VTE) was $25 \%$, and $10 \%$ of the cohort with VTE, died (33). In a case series of 3 patients presenting with bilateral lower limb ischemia and cerebral infarcts, antiphospholipid antibodies were detected, implicating a possible role in the thrombotic events (36). These antibodies, as well as other coagulation markers, may become detectable, in response to severe infections and critical illness $(34,36)$. In a cohort of 1,026 patients, Wang et al. reported that $40 \%$ had an elevated thrombotic risk, and without prophylaxis, $11 \%$ developed VTE (37). COVID-19 related thrombotic complications include arterial thromboembolism, deep vein thrombosis, intracardiac thrombus, PE, and stroke (37). The anticoagulation regimen of COVID-19 patients' needs to be tailored individually, and has to be balanced against potential bleeding complications associated with thrombotic prophylaxis (37).

\section{Hyperinflammatory shock in children}

The number of children affected by COVID-19 remains low around the world. In the US and the UK, only $1.7 \%$ and $2 \%$ of the positive cases, respectively, occurred in patients younger than 18 (38). However, there are emerging reports of an atypical hyperinflammatory syndrome in children (39). The symptoms include persistent fever, exanthema, lymphadenopathy, conjunctival injection, and other changes in the mucosae, raising the concern of COVID-19 as a trigger for Kawasaki disease $(\mathrm{KW})$, or a Kawasaki-like syndrome $(38,40)$. $\mathrm{KW}$ is a systemic vasculitis of childhood, with unknown etiology, which may seriously compromise the cardiovascular system $(38,40)$, including long-term complications such as coronary artery aneurysms (38). Previous studies linked the disease with viral infections in the previous 30 days of presentation in $9-42 \%$ of the cases (40). The identification of different strains of the coronavirus family as a potential etiology has also been described following outbreaks $(40,41)$. It is essential to highlight that these cases remain rare, and that children with COVID-19 usually present as asymptomatic cases or have 
mild symptoms $(38,40)$.

\section{Antiviral drugs}

Lopinavir/Ritonavir is an antiretroviral drug combination (42). It works by inhibiting the viral RNA replication, and has shown in vitro activity against SARS-CoV $(43,44)$. However, Cao et al. reported showed no difference between those treated with lopinavir/ritonavir versus standard of care (SOC) in 199 COVID-19 patients [hazard ratio (HR) for clinical improvement: 1.24; $95 \%$ CI, 0.90 to 1.72] (42). In terms of 28-day mortality, lopinavir/ritonavir group was not statistically different from SOC (19.2\% vs. $25.0 \%$; difference, -5.8 percentage points; $95 \%$ CI, -17.3 to 5.7 ) (42). Cardiovascular adverse events are notable. Conduction disturbances, QT interval prolongation (especially combined with other QTprolonging medications, and electrolyte disturbances), and PR prolongation (of particular risk in patients with structural heart disease, pre-existing conduction system abnormalities, and ischemic heart disease), have been reported, as has hyperlipidemia $(42,45)$.

Remdesivir is a novel drug, which works by inhibiting the RNA-dependent RNA polymerase (46). Different research groups are studying its efficacy in COVID-19, because it demonstrated inhibition of SARS and MERS (46). A randomized controlled trial (RCT) involving 385 subjects from 10 centers in China demonstrated a numerical reduction in time to clinical improvement in those treated earlier, however this was not statistically significant [HR 1.23 (95\% CI, 0.87-1.75)] (42). In contrast, Grein et al. studied the compassionate use of remdesivir in 53 patients, observing an improvement in oxygen support in $68 \%$ of the patients, extubation in $57 \%$ of the patients on mechanical ventilation, and the cessation of ECMO in $75 \%$ of the patients (47). Beigel et al. conducted a double-blind, randomized, placebocontrolled trial in 1,059 patients (538 assigned to remdesivir and 521 to placebo) (48). There was a shortening in the recovery time from 15 to 11 days for the remdesivir group (rate ratio for recovery: $1.32 ; 95 \% \mathrm{CI}, 1.12-1.55 ; \mathrm{P}<0.001$ ). The National Institutes of Health released preliminary data from this clinical trial (\#NCT04280705), and on May 1st, the Food and Drug Administration (FDA) approved the use of this drug in severe COVID-19 patients (49).

Regarding the optimal duration of the antiviral, a randomized, open-label, phase 3 trial was conducted in 397 patients (50). After adjusting for baseline clinical status, no significant difference between 5 vs. 10 days of treatment was reported $(\mathrm{P}=0.14)(50)$. There was no placebo control group, limiting the interpretation of the overall benefit of remdesivir (50). Specific cardiovascular toxicities of this therapy are not yet clear.

\section{Cbloroquine/bydroxychloroquine (CQ/HCQ)}

These are antimalarial drugs (51). Theoretically, they could inhibit viral replication by increasing endosomal $\mathrm{pH}$ since the virus requires low $\mathrm{pH}$ to thrive, thus impairing the release of the genetic material, inhibiting replication (52). The available data are inconclusive. Two studies currently in pre-print reported conflicting results. A Chinese study involving 30 patients receiving HCQ reported high viral clearance (52). In comparison, an American study categorizing 368 hospitalized veterans into 3 groups [HCQ, HCQ + Azithromycin (AZ), and those unexposed to HCQ], demonstrated no significant benefit in mortality $27.8 \%$ in the $\mathrm{HC}$ group, $22.1 \%$ in the $\mathrm{HC}+\mathrm{AZ}$ group, and $11.4 \%$ in the unexposed group) (53). In a multicenter Chinese RCT involving 150 patients, there were no statistically significant differences in the 28-day negative conversion rate of SARSCoV-2 between the HCQ and SOC versus SOC groups ( $85.4 \%$ vs. $81.3 \%, \mathrm{P}=0.341$ ) (54). However, a possible beneficial effect on clinical symptoms (HR, 8.83; 95\% CI, 1.09-71.3), and a significant reduction in CRP [6.986 (HCQ+SOC) vs. 2.723 (SOC) milligram/liter, $\mathrm{P}=0.045$ ] were reported (54). The most common cardiovascular effects are QT prolongation and conduction disturbances (atrioventricular block and bundle branch block) (52). Other long-term adverse effects include cardiomyopathy (restrictive or dilated), and congestive HF (51).

\section{Camostat mesylate}

This is a serine protease inhibitor approved in Japan for chronic pancreatitis and reflux esophagitis (55). The interaction between membrane-bound ACE2 and S protein to enter the host cells is dependent on TMPRSS2 (10). A clinical trial is underway, investigating the potential of camostat mesylate for treating COVID-19 (56).

\section{Monoclonal antibodies and convalescent plasma}

In a preliminary uncontrolled study of five critically ill patients, the administration of convalescent plasma containing neutralizing antibody showed improvement (57). Any potential cardiovascular adverse effects are yet to be reported. 


\section{Vaccine}

$\mathrm{B}$ cell and $\mathrm{T}$ cell epitopes derived from the $\mathrm{S}$ and $\mathrm{N}$ proteins in SARS-CoV-2 sequences, are being studied (58). Targeting these epitopes may potentially confer immunity against COVID-19 (58). The first clinical testing in humans of the COVID-19 vaccine was on March 16, 2020 (59). One hundred and fifteen vaccine candidates were available as of April 8, 2020 (59). As of September 3 2020, there was a 2.5 -fold increase of vaccines candidates, and 33 of those 321 are currently in phase III clinical trials (60). Potential candidates include messenger RNA vaccine, mRNA-1273 from Moderna, Ad5-nCoV from CanSino Biologicals, INO-4800 from Inovio, LV-SMENP-DC and pathogenspecific aAPC from Shenzhen Geno-Immune Medical Institute, and ChAdOx1 from Jenner Institute, University of Oxford. Adjuvant vaccine, which works by enhancing immunogenicity, is also being investigated (59).

\section{Role of angiotensin receptor inbibitors/angiotensin receptor blockers (ACEi/ARBs)}

Conflicting reports speculated a possible link between ACEi/ ARBs and more severe COVID-19 cases (61). The concern arises from the theoretical overexpression of membranebound ACE2, predisposing to viral entry and propagation of SARS-CoV2 (12). On the contrary, several studies demonstrated a protective role of membrane-bound ACE2 and angiotensin in lung injury models (61). In viral pneumonia, it has shown a beneficial effect in reducing the pulmonary inflammatory response and cytokine release $(61,62)$. To date, major international societies, including the American College of Cardiology (ACC), American Heart Association (AHA), Heart Failure Society of America (HFSA), and the European Society of Cardiology (ESC), recommend continuing treatment with ACEi /ARBs in patients already taking these therapies $(62,63)$. Sudden cessation of ACEi/ARBs in highrisk patients could potentially lead to significant cardiac decompensation (61). Mehta et al. studied the association of ACEi/ARB use and COVID-19 test positivity. Out of 18,472 patients tested for COVID-19, 1735 (9.4\%) had a positive test, and $12.4 \%$ were on either ACEi or ARB (64). No significant association was found between ACEi or ARB use and COVID-19 test positivity (overlap propensity score-weighted OR: 0.97; 95\% CI, 0.81-1.15) (64). Selected experimental therapies being studied in clinical trials are shown in Table 1.

Healthcare workers (HCWs) are at increased risk of COVID-19, and infections continue to be on the rise worldwide. Zhou et al. reported hospital-associated transmission to HCWs in China, and as of February 11, 2020, there were 3019 reported cases of infections in Chinese HCWs and at least 22 deaths (66). To effectively battle the pandemic, specific measures need to be followed in the cardiovascular community.

\section{Cardiac catbeterization laboratory and COVID-19}

The ACC, the ESC, and the Society for Cardiovascular Angiography and Interventions (SCAI), recommend deferring elective procedures in the pandemic, including diagnostic cardiac catheterization, percutaneous coronary intervention (PCI) for stable ischemic heart disease, and non-essential structural heart interventions $(67,68)$. The purpose of this is to minimize patient and HCWs exposure to COVID-19 including asymptomatic carriers, and maximize the availability of inpatient beds and personal protective equipment (PPE). However, in all patients with suspected or confirmed COVID-19, it is paramount to wear appropriate PPE, including gown, gloves, goggles (face shields), and N95 masks (68). In patients with borderline respiratory status, consideration should be given to intubation prior to arrival to the catheterization laboratory, and non-invasive positive pressure ventilation [Continuous positive Airway Pressure (CPAP) and Bi-level Positive Airway Pressure (BiPAP)] should be avoided, given the risk of aerosolization and provider exposure. While fibrinolysis can be considered in patients with STEMI and confirmed COVID-19 infection, primary PCI should still be the treatment of choice if appropriate PPE can be utilized $(68,69)$. In low-risk patients with type II nonST-elevation myocardial infarction (NSTEMI) and confirmed COVID-19, medical management may be considered initially. Nevertheless, in clinically unstable patients with non STelevation acute coronary syndrome (NSTE-ACS), cardiac catheterization with PCI should be performed, aiming for rapid discharge $(68,69)$. While waiting for confirmatory testing for COVID-19 is not recommended for patients with suspected STEMI, in medically stable patients with NSTEACS, it may be worthwhile to obtain confirmatory testing in patients with suspected COVID-19 $(68,69)$. Patients with critical aortic stenosis or severe, symptomatic aortic stenosis (AS) should still proceed with transcatheter aortic valve replacement (TAVR) in a timely fashion. However, consideration should be given to deferring patients with severe AS who are not symptomatic or minimally symptomatic until the hospital can safely accommodate such patients. 
Table 1 Potential treatment options being studied for COVID-19 and their cardiovascular adverse effects (65)

\begin{tabular}{|c|c|c|c|}
\hline Drug & ClinicalTrial.gov identifier & Mechanism of action & CV side effects \\
\hline Tocilizumab & NCT04320615 & $\begin{array}{l}\text { IL-6 receptor antagonist. } \\
\text { Modulates immune response }\end{array}$ & Hyperlipidemia \\
\hline Camostat mesylate & NCT04321096 & Inhibits viral entry & No CV effect described \\
\hline Sarilumab & NCT04315298 & $\begin{array}{l}\text { IL-6 receptor antagonist. } \\
\text { Modulates immune response }\end{array}$ & Hypertriglyceridemia \\
\hline Fingolimod & NCT04280588 & $\begin{array}{l}\text { Sphingosine-1 phosphate } \\
\text { modulator. Reduces lymphocyte } \\
\text { migration. Modulates immune } \\
\text { response }\end{array}$ & $\begin{array}{l}\text { Bradyarrhythmia and AV block, } \\
\text { hypertension, QT prolongation }\end{array}$ \\
\hline Eculizumab & NCT04288713 & $\begin{array}{l}\text { Blocks complement protein C5 by } \\
\text { inhibiting cleavage to C5a to C5b. } \\
\text { Modulates immune response }\end{array}$ & Hypertension, peripheral edema \\
\hline Bevacizumab & NCT04305106 NCT04275414 & $\begin{array}{l}\text { Inhibits VEGF, reducing vascular } \\
\text { permeability and pulmonary } \\
\text { edema. Modulates immune } \\
\text { response }\end{array}$ & $\begin{array}{l}\text { Direct myocardial toxicity } \\
\text { vs. exacerbation of CV } \\
\text { disease, hypertension, arterial } \\
\text { thromboembolic events }\end{array}$ \\
\hline Interferon & $\begin{array}{l}\text { EUCTR2020-001023-14-GB NCT04293887 } \\
\text { ChiCTR2000030013 }\end{array}$ & $\begin{array}{l}\text { Activation of immunological } \\
\text { response }\end{array}$ & $\begin{array}{l}\text { Direct myocardial toxicity vs. } \\
\text { exacerbation of CV disease, } \\
\text { chest pain, edema, arrhythmia }\end{array}$ \\
\hline Interferon & NCT04322682 & $\begin{array}{l}\text { Inhibits inflammasome NLP3 and } \\
\text { microtubule formation, limiting } \\
\text { the myocardial necrosis and } \\
\text { pneumonia development }\end{array}$ & No CV effect described \\
\hline Pirfenidone & NCT04282902 ChiCTR2000030333 & $\begin{array}{l}\text { Inhibits TGF-beta and TNF-alpha. } \\
\text { Modulates immune response and } \\
\text { reduces pulmonary fibrosis }\end{array}$ & No CV effect described \\
\hline Favipiravir & NCT04310228ChiCTR2000030987 & Inhibition viral RNA polymerase & No CV effect described \\
\hline
\end{tabular}

RNA, ribonucleic acid; CV, cardiovascular; AVB, atrioventricular block; BBB, bundle branch block; HF, heart failure; IL, interleukin; TNF, tumor necrosis factor; VEGF, vascular endothelial growth factor; PMNs, polymorphonuclear leukocytes; TGF, transforming growth factor. 


\section{Echocardiography laboratory and COVID-19}

The American Society of Echocardiography (ASE) and the European Association of Cardiovascular Imaging (EACVI) recommend that during the pandemic transthoracic echocardiography (TTE), transesophageal echocardiography (TEE), and stress echocardiography should be deferred if not urgent/emergent $(70,71)$. Strict droplet precautions must occur in echocardiography laboratories/cardiac catheterization laboratories/ electrophysiology laboratory, wards, or emergency departments (EDs) while performing an imaging test in patients with suspected/confirmed COVID-19 (71). In ICUs, operating rooms (ORs), and with ventilated patients (invasive or non-invasive), airborne precautions are necessary (71). TEE warrants special attention because of the increased risk of spreading SARS-CoV2 via aerosolization that may be associated with coughing/ gagging during the procedure (71). Adjuvant imaging modalities such as computed tomography (CT) should be considered as an alternative to TEE when feasible (e.g., exclusion of left atrial appendage thrombus before cardioversion) (71). For TTE or stress echocardiography in patients without COVID-19, standard precautions should be implemented (71). With the need to provide rapid, bedside testing, cardiac point-of-care ultrasound (POCUS) has gained increasing attention, given its portability (71). ASE and EACVI have created a cardiac POCUS protocol for COVID-19 (70,71).

Additionally, the minimum number of HCWs should be involved in the acquisition of imaging data, with a review of previous study images where available, and performance of targeted examinations to answer the clinical questions, and where available, to use dedicated machines/laboratories for COVID-19 patients (71). If possible, procedures on the suspected/confirmed COVID-19 patients should be performed at the end of the day (to minimize delays in cleaning) and utilizing negative-pressure rooms or highefficiency particulate (HEPA) filters if available (71). It is of utmost importance for the HCWs to practice correct PPE donning and doffing techniques (71). Table 2 lists the effective use of PPE while performing various cardiovascular procedures.

\section{Invasive and non-invasive electrophysiology procedures and COVID-19}

The Heart Rhythm Society (HRS) recommends delaying non- urgent/elective electrophysiology (EP) procedures (72). For cases that would cause a threat to patient' lives, permanent dysfunction to an organ-extremity, or imminent deterioration to severe status, EP procedures should be done with appropriate PPE, adhering to the same recommendations as for other urgent procedures described above $(67,72)$.

\section{Cardiac arrest and cardiopulmonary resuscitation and COVID-19}

During cardiac arrest of a suspected COVID-19 patient, according to the American Heart Association (AHA), cardiopulmonary resuscitation (CPR) must be performed with appropriate PPE (N95 mask, long-sleeve gown, gloves, and face shield) $(72,73)$. Oxygenation/ventilation strategies should be prioritized to minimize aerosolization, and early endotracheal intubation should be performed when possible (73). Immediate defibrillation of a shockable rhythm provides less risk of aerosolization than compressions in a patient with an unsecured airway (73). If early resuscitation is unsuccessful, continuing with the maneuvers may be futile, and poses high-risk to the rescuer in the absence of adequate PPE (73). It is essential to consider the likelihood of success versus risks for the patients and the resuscitation team (73).

\section{Management of critically ill patients and COVID-19}

Other non-ICU HCWs may be called upon to assist critically ill patients on the front-line. Healthcare resources are of supreme importance in this pandemic. Cardiac ICUs and ORs may be transformed into medical ICUs due to the increasing demand for mechanical ventilation (74). A transition of inpatient wards and other facilities into dedicated COVID-19 units in anticipation of the surge in hospitalization has been observed (74). Likewise, staffing models in cardiac ICUs might need to be modified to allow for tiered oversight of non-critical care trained physicians (74). It is increasingly important to work as a team and utilize the available resources appropriately, while minimizing the exposure and spread of the infection.

\section{COVID-19 and the rise of virtual cardiology}

Telemedicine incorporating appropriate use of web portals, computers, and smartphones, is another valuable way to minimize the risk of infection. It allows the patients to be 
Table 2 Personal protective equipment (PPE) recommendations for COVID-19, while performing various cardiovascular procedures

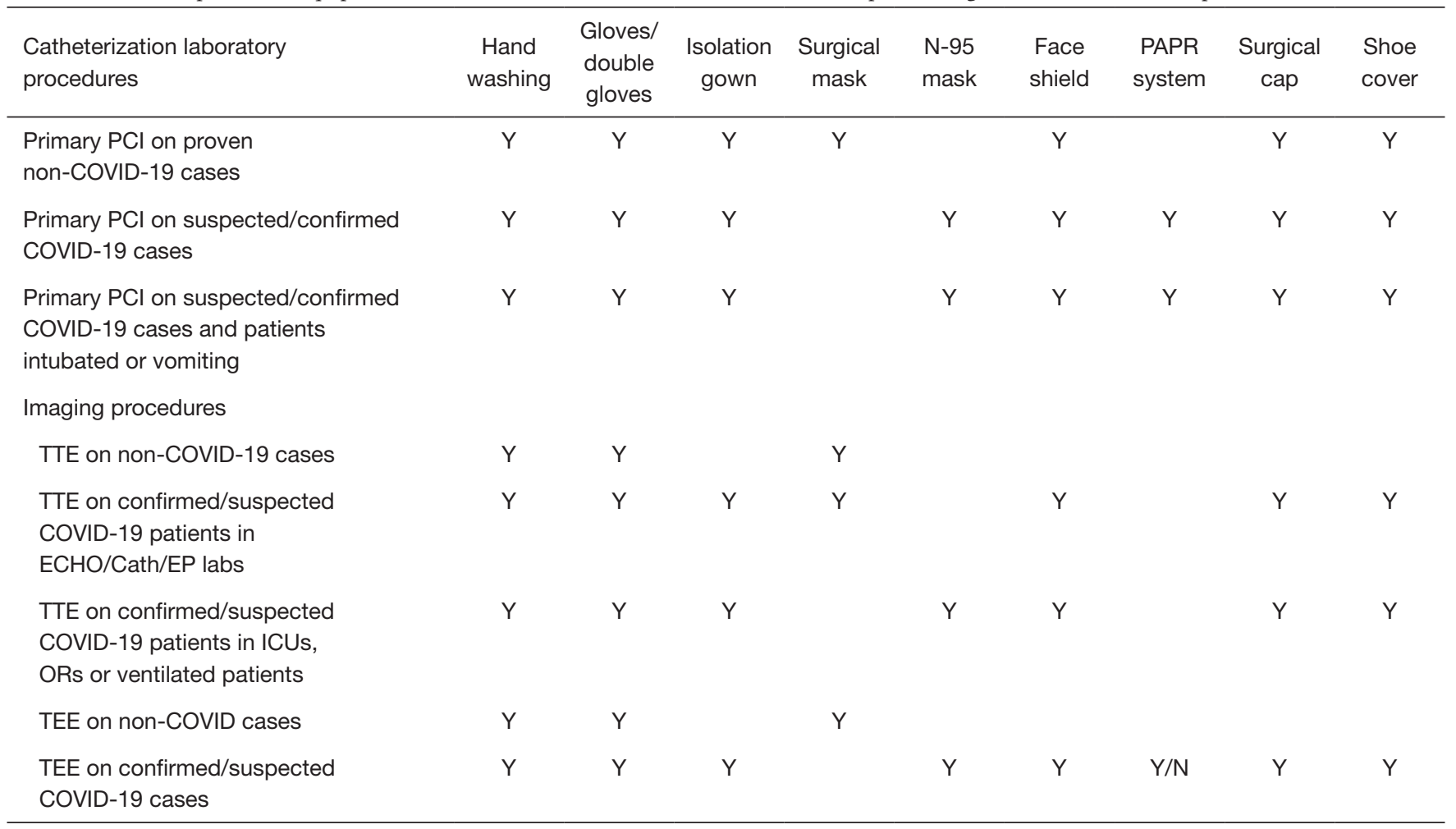

$\mathrm{PCl}$, percutaneous coronary intervention; TTE, transthoracic echocardiogram; ECHO, echocardiography; Cath, cardiac catheterization; EP, electrophysiology; Labs, laboratories; ICUs, intensive care units; ORs, operating rooms.

assessed while reducing the exposure of both the HCW and the patients (75). Virtual visits consultations during the pandemic are essential in containing the spread of COVID-19, while maintaining optimal cardiovascular care. Trainees should also be involved in telemedicine, with the transition of education to a virtual platform (75). Major societies should be applauded for transforming international conferences, including the ACC and ASE annual scientific meetings, to a virtual online platform (76). This unprecedented effort serves well to maintain ongoing research collaboration and education for cardiovascular professionals worldwide.

\section{Conclusions}

COVID-19 has important cardiovascular implications, potentially mediated through several mechanisms, including direct myocardial injury, worsening of pre-existing cardiovascular co-morbidities, and adverse cardiovascular effects of potential therapies for COVID-19. Multiple studies of novel therapeutics are underway. A spotlight has been cast on virtual cardiology, as the cardiovascular community embraces international collaboration to combat COVID-19 and mitigate its impact on the cardiovascular system.

\section{Acknowledgments}

The authors would like to thank Dave Schumick, BS, CMI, and Joseph Pangrace, BFA, CMI for expert assistance with graphic illustrations. The graphics are reprinted with the permission of the Cleveland Clinic Center for Medical Art \& Photography (C) 2020. All Rights Reserved.

Funding: None.

\section{Footnote}

Provenance and Peer Review: This article was commissioned by the editorial office, Cardiovascular Diagnosis and Therapy for the series "Heart Valve Disease". The article has undergone external peer review. 
Reporting Checklist: The authors have completed the Narrative Review reporting checklist. Available at http:// dx.doi.org/10.21037/cdt-20-779

Conflicts of Interest: All authors have completed the ICMJE uniform disclosure form (available at http://dx.doi. org/10.21037/cdt-20-779). The series "Heart Valve Disease" was commissioned by the editorial office without any funding or sponsorship. BX served as the unpaid Guest Editor of the series. Dr. LGS reports other from Posthorax $\mathrm{GmbH}$, outside the submitted work. The authors have no other conflicts of interest to declare.

Ethical Statement: The authors are accountable for all aspects of the work in ensuring that questions related to the accuracy or integrity of any part of the work are appropriately investigated and resolved.

Open Access Statement: This is an Open Access article distributed in accordance with the Creative Commons Attribution-NonCommercial-NoDerivs 4.0 International License (CC BY-NC-ND 4.0), which permits the noncommercial replication and distribution of the article with the strict proviso that no changes or edits are made and the original work is properly cited (including links to both the formal publication through the relevant DOI and the license). See: https://creativecommons.org/licenses/by-nc-nd/4.0/.

\section{References}

1. World Health Organization. WHO Timeline COVID-19 [Internet]. 2020 [cited 2020 Sept 30]. Available online: https://www.who.int/news-room/detail/08-042020-who-timeline---covid-19

2. Zhou P, Yang XL, Wang XG, et al. A pneumonia outbreak associated with a new coronavirus of probable bat origin. Nature 2020;579:270-3.

3. Center for Systems Science and Engineering (CSSE) at Johns Hopkins University (JHU). COVID-19 Dashboard [Internet].2020 [cited 2020 Sept 30]. Available online: https://coronavirus.jhu.edu/map.html

4. Li X, Geng M, Peng Y, et al. Molecular immune pathogenesis and diagnosis of COVID-19. J Pharm Anal 2020;10:102-8.

5. Clerkin KJ, Fried JA, Raikhelkar J, et al. COVID-19 and Cardiovascular Disease. Circulation 2020;141:1648-55.

6. Centers for Disease Control and Prevention (CDC). Public Health Recommendations for Community-Related
Exposure [Internet] 2020. [cited 2020 Apr 8]. Available online: https://www.cdc.gov/coronavirus/2019-ncov/php/ public-health-recommendations.html

7. Aylward B, Liang W. Report of the WHO-China Joint Mission on Coronavirus Disease 2019 (COVID-19). WHO 2020;2019:16-24.

8. Huang C, Wang Y, Li X, et al. Clinical features of patients infected with 2019 novel coronavirus in Wuhan, China. Lancet 2020;395:497-506.

9. Yan R, Zhang Y, Li Y, et al. Structural basis for the recognition of SARS-CoV-2 by full-length human ACE2. Science 2020;367:1444-8.

10. Zhao Y, Zhao Z, Wang Y, et al. Single-Cell RNA Expression Profiling of ACE2, the Receptor of SARSCoV-2. Am J Respir Crit Care Med 2020;202:756-9.

11. Channappanavar R, Zhao J, Perlman S. T cell-mediated immune response to respiratory coronaviruses. Immunol Res 2014;59:118-28.

12. Zhang H, Penninger JM, Li Y, et al. Angiotensinconverting enzyme 2 (ACE2) as a SARS-CoV-2 receptor: molecular mechanisms and potential therapeutic target. Intensive Care Med 2020;46:586-90.

13. Zhou F, Yu T, Du R, et al. Clinical course and risk factors for mortality of adult inpatients with COVID-19 in Wuhan, China: a retrospective cohort study. Lancet 2020;395:1054-62.

14. Iacobucci G. Covid-19: all non-urgent elective surgery is suspended for at least three months in England. BMJ 2020;368:m1106.

15. Shi S, Qin M, Shen B, et al. Association of Cardiac Injury with Mortality in Hospitalized Patients With COVID-19 in Wuhan, China. JAMA Cardiol 2020;5:802-10.

16. Booth CM, Matukas LM, Tomlinson GA, et al. Clinical features and short-term outcomes of 144 patients with SARS in the greater Toronto area. JAMA 2003;289:2801-9.

17. Badawi A, Ryoo SG. Prevalence of comorbidities in the Middle East respiratory syndrome coronavirus (MERS$\mathrm{CoV}$ ): a systematic review and meta-analysis. Int J Infect Dis 2016;49:129-33.

18. Ye J, Rust G, Baltrus P, et al. Cardiovascular risk factors among Asian Americans: results from a National Health Survey. Ann Epidemiol 2009;19:718-23.

19. Arentz M, Yim E, Klaff L, et al. Characteristics and Outcomes of 21 Critically Ill Patients With COVID-19 in Washington State. JAMA 2020;323:1612-4.

20. Docherty AB, Harrison EM, Green CA, et al. Features of 20133 UK patients in hospital with covid-19 using the ISARIC WHO Clinical Characterisation 
Protocol: prospective observational cohort study. BMJ 2020;369:m1985.

21. Wang D, Hu B, Hu C, et al. Clinical Characteristics of 138 Hospitalized Patients With 2019 Novel CoronavirusInfected Pneumonia in Wuhan, China. JAMA 2020;323:1061-9.

22. Lippi G, Lavie CJ, Sanchis-Gomar F. Cardiac troponin I in patients with coronavirus disease 2019 (COVID-19): Evidence from a meta-analysis. Prog Cardiovasc Dis 2020;63:390-1.

23. Alhogbani T. Acute myocarditis associated with novel Middle East Respiratory Syndrome Coronavirus. Ann Saudi Med 2016;36:78-80.

24. Hu H, Ma F, Wei X, et al. Coronavirus fulminant myocarditis saved with glucocorticoid and human immunoglobulin. Eur Heart J 2021;42:206.

25. Kwong JC, Schwartz KL, Campitelli MA, et al. Acute Myocardial Infarction after Laboratory-Confirmed Influenza Infection. N Engl J Med 2018;378:345-53.

26. Zeng JH, Liu YX, Yuan J, et al. First case of COVID-19 complicated with fulminant myocarditis: a case report and insights. Infection 2020;48:773-7.

27. Liu K, Fang YY, Deng Y, et al. Clinical characteristics of novel coronavirus cases in tertiary hospitals in Hubei Province. Chin Med J (Engl) 2020;133:1025-31.

28. Aslam S, Mehra MR. COVID-19: Yet another coronavirus challenge in transplantation. J Heart Lung Transplant 2020;39:408-9.

29. Ramanathan K, Antognini D, Combes A, et al. Planning and provision of ECMO services for severe ARDS during the COVID-19 pandemic and other outbreaks of emerging infectious diseases. Lancet Respir Med 2020;8:518-26.

30. Shekar K, Badulak J, Peek G, et al. Extracorporeal Life Support Organization COVID-19 Interim Guidelines. ASAIO J 2020;66:707-21.

31. Hendren NS, Drazner MH, Bozkurt B, et al. Description and Proposed Management of the Acute COVID-19 Cardiovascular Syndrome. Circulation 2020;141:1903-14.

32. MacLaren G, Fisher D, Brodie D. Preparing for the Most Critically Ill Patients With COVID-19: The Potential Role of Extracorporeal Membrane Oxygenation. JAMA 2020;323:1245-6.

33. Cui S, Chen S, Li X, et al. Prevalence of venous thromboembolism in patients with severe novel coronavirus pneumonia. J Thromb Haemost 2020;18:1421-4.

34. Bikdeli B, Madhavan MV, Jimenez D, et al. COVID-19 and Thrombotic or Thromboembolic Disease:
Implications for Prevention, Antithrombotic Therapy, and Follow-Up: JACC State-of-the-Art Review. J Am Coll Cardiol 2020;75:2950-73.

35. Poissy J, Goutay J, Caplan M, et al. Pulmonary Embolism in Patients With COVID-19: Awareness of an Increased Prevalence. Circulation 2020;142:184-6.

36. Zhang Y, Xiao M, Zhang S, et al. Coagulopathy and Antiphospholipid Antibodies in Patients with Covid-19. N Engl J Med 2020;382:e38.

37. Wang T, Chen R, Liu C, et al. Attention should be paid to venous thromboembolism prophylaxis in the management of COVID-19. Lancet Haematol 2020;7:e362-3.

38. Viner RM, Whittaker E. Kawasaki-like disease: emerging complication during the COVID-19 pandemic. Lancet 2020;395:1741-3.

39. Riphagen S, Gomez X, Gonzalez-Martinez C, et al. Hyperinflammatory shock in children during COVID-19 pandemic. Lancet 2020;395:1607-8.

40. Jones VG, Mills M, Suarez D, et al. COVID-19 and Kawasaki Disease: Novel Virus and Novel Case. Hosp Pediatr 2020;10:537-40.

41. Esper F, Shapiro ED, Weibel C, et al. Association between a novel human coronavirus and Kawasaki disease. J Infect Dis 2005;191:499-502.

42. Cao B, Wang Y, Wen D, et al. A Trial of LopinavirRitonavir in Adults Hospitalized with Severe Covid-19. N Engl J Med 2020;382:1787-99.

43. Chu CM, Cheng VC, Hung IF, et al. Role of lopinavir/ ritonavir in the treatment of SARS: initial virological and clinical findings. Thorax 2004;59:252-6.

44. Elfiky AA. Anti-HCV, nucleotide inhibitors, repurposing against COVID-19. Life Sci 2020;248:117477.

45. Driggin E, Madhavan MV, Bikdeli B, et al. Cardiovascular Considerations for Patients, Health Care Workers, and Health Systems During the COVID-19 Pandemic. J Am Coll Cardiol 2020;75:2352-71.

46. Wang M, Cao R, Zhang L, et al. Remdesivir and chloroquine effectively inhibit the recently emerged novel coronavirus (2019-nCoV) in vitro. Cell Res 2020;30:269-71.

47. Grein J, Ohmagari N, Shin D, et al. Compassionate Use of Remdesivir for Patients with Severe Covid-19. N Engl J Med 2020;382:2327-36.

48. Beigel JH, Tomashek KM, Dodd LE, et al. Remdesivir for the Treatment of Covid-19 - Final Report. N Engl J Med 2020;383:1813-26.

49. Dolin R, Hirsch MS. Remdesivir - An Important First Step. N Engl J Med 2020;383:1886-7.

50. Goldman JD, Lye DCB, Hui DS, et al. Remdesivir for 5 
or 10 Days in Patients with Severe Covid-19. N Engl J Med 2020;383:1827-37.

51. Tonnesmann E, Kandolf R, Lewalter T. Chloroquine cardiomyopathy - a review of the literature. Immunopharmacol Immunotoxicol 2013;35:434-42.

52. Gao J, Tian Z, Yang X. Breakthrough: Chloroquine phosphate has shown apparent efficacy in treatment of COVID-19 associated pneumonia in clinical studies. Biosci Trends 2020;14:72-3.

53. Magagnoli J, Narendran S, Pereira F, et al. Outcomes of hydroxychloroquine usage in United States veterans hospitalized with Covid-19. medRxiv 2020. doi: 10.1016/ j.medj.2020.06.001

54. Tang W, Cao Z, Han M, et al. Hydroxychloroquine in patients with mainly mild to moderate coronavirus disease 2019: open label, randomised controlled trial. BMJ 2020;369:m1849.

55. Gibo J, Ito T, Kawabe K, et al. Camostat mesilate attenuates pancreatic fibrosis via inhibition of monocytes and pancreatic stellate cells activity. Lab Invest 2005;85:75-89.

56. U.S. National Library of Medicine. The Impact of Camostat Mesilate on COVID-19 Infection (CamoCO-19) [Internet]. 2020 [cited 2020 Apr 15]. Available online: https:/clinicaltrials.gov/ct2/show/NCT04321096

57. Shen C, Wang Z, Zhao F, et al. Treatment of 5 Critically Ill Patients With COVID-19 With Convalescent Plasma. JAMA 2020;323:1582-9.

58. Ahmed SF, Quadeer AA, McKay MR. Preliminary Identification of Potential Vaccine Targets for the COVID-19 Coronavirus (SARS-CoV-2) Based on SARSCoV Immunological Studies. Viruses 2020;12:254.

59. Thanh Le T, Andreadakis Z, Kumar A, et al. The COVID-19 vaccine development landscape. Nat Rev Drug Discov 2020;19:305-6.

60. Thanh Le T, Cramer JP, Chen R, et al. Evolution of the COVID-19 vaccine development landscape. Nat Rev Drug Discov 2020;19:667-8.

61. Kuba K, Imai Y, Rao S, et al. A crucial role of angiotensin converting enzyme 2 (ACE2) in SARS coronavirusinduced lung injury. Nat Med 2005;11:875-9.

62. DeCarolis DD, Chen YC, Westanmo AD. Evaluation of a Potential Interaction Between New Regimens to Treat Hepatitis C and Warfarin: Twelve-Week Post-treatment Follow-up. Ann Pharmacother 2017;51:439-40.

63. Adams JG, Walls RM. Supporting the Health Care Workforce During the COVID-19 Global Epidemic. JAMA 2020;323:1439-40.

64. Mehta N, Kalra A, Nowacki AS, et al. Association of
Use of Angiotensin-Converting Enzyme Inhibitors and Angiotensin II Receptor Blockers With Testing Positive for Coronavirus Disease 2019 (COVID-19). JAMA Cardiol 2020;5:1020-6.

65. U.S. National Library of Medicine. 2020 Clinical Trials Related to COVID-19 Listed on ClinicalTrials.gov. [Internet]. 2020 [cited 2020 Sept 14]. Available online: https://clinicaltrials.gov/ct2/results?cond=COVID-19

66. Zhou P, Huang Z, Xiao Y, et al. Protecting Chinese healthcare workers while combating the 2019 novel coronavirus. Infect Control Hosp Epidemiol 2020;41:745-6.

67. European Society of Cardiology. ESC Guidance for the Diagnosis and Management of CV Disease during the COVID-19 Pandemic [Internet]. 2020 [cited 2020 May 1]. p. 1-105. Available online: https://www.escardio.org/ Education/COVID-19-and-Cardiology/ESC-COVID-19Guidance

68. Welt FGP, Shah PB, Aronow HD, et al. Catheterization Laboratory Considerations During the Coronavirus (COVID-19) Pandemic: From the ACC's Interventional Council and SCAI. J Am Coll Cardiol 2020;75:2372-5.

69. Jing ZC, Zhu HD, Yan XW, et al. Recommendations from the Peking Union Medical College Hospital for the management of acute myocardial infarction during the COVID-19 outbreak. Eur Heart J 2020;41:1791-4.

70. Skulstad H, Cosyns B, Popescu BA, et al. COVID-19 pandemic and cardiac imaging: EACVI recommendations on precautions, indications, prioritization, and protection for patients and healthcare personnel. Eur Heart J Cardiovasc Imaging 2020;21:592-8.

71. Johri AM, Galen B, Kirkpatrick JN, et al. ASE Statement on Point-of-Care Ultrasound during the 2019 Novel Coronavirus Pandemic. J Am Soc Echocardiogr 2020;33:670-3.

72. Lakkireddy DR, Chung MK, Gopinathannair R, et al. Guidance for cardiac electrophysiology during the COVID-19 pandemic from the Heart Rhythm Society COVID-19 Task Force; Electrophysiology Section of the American College of Cardiology; and the Electrocardiography and Arrhythmias Committee of the Council on Clinical Cardiology, American Heart Association. Heart Rhythm 2020;17:e233-e41.

73. Edelson DP, Sasson C, Chan PS, et al. Interim Guidance for Basic and Advanced Life Support in Adults, Children, and Neonates With Suspected or Confirmed COVID-19: From the Emergency Cardiovascular Care Committee and Get With The Guidelines-Resuscitation Adult and Pediatric Task Forces of the American Heart Association. 
Circulation 2020;141:e933-43.

74. Katz JN, Sinha SS, Alviar CL, et al. COVID-19 and Disruptive Modifications to Cardiac Critical Care Delivery: JACC Review Topic of the Week. J Am Coll Cardiol 2020;76:72-84.

75. Hollander JE, Carr BG. Virtually Perfect? Telemedicine

Cite this article as: Brizneda MV, Bansal A, Jain V, Kapadia S, Svensson LG, Menon V, Cremer P, Reed G, Rampersad P, Grimm R, Griffin BP, Xu B. Coronavirus disease and the cardiovascular system: a narrative review of the mechanisms of injury and management implications. Cardiovasc Diagn Ther 2021;11(3):939-953. doi: 10.21037/cdt-20-779 for Covid-19. N Engl J Med 2020;382:1679-81.

76. American College of Cardiology. ACC.20/WCC in Chicago is Canceled. [Internet]. 2020 [cited 2020 Sept 14]. Available online: https://www.acc.org/about-acc/ press-releases/2020/03/09/08/43/acc20-wcc-in-chicago-iscanceled 